\title{
Visualização de contratos eletrônicos: uma proposta de artefato com base em Proposições de Design
}

\section{Electronic contract visualization: an artifact proposal based on Design Propositions}

\author{
Renata Zappelli Marzullo, Universidade Federal do Rio de Janeiro \\ rvzappelli@gmail.com
}

\author{
André Ribeiro de Oliveira, Escola Superior de Desenho Industrial da UERJ \\ andre.ribeiro@eng.uerj.br
}

\author{
André Soares Monat, Escola Superior de Desenho Industrial da UERJ \\ andresmonat@yahoo.com.br
}

\section{Resumo}

As relações comerciais assumiram novas caraterísticas com a Internet que possibilitaram o comércio virtual regido por contratos eletrônicos como mediadores dos direitos e obrigações entre usuários e empresas. As recomendações de isolamento social, devido à pandemia da COVID-19, têm impulsionado ainda mais o comércio eletrônico. Entretanto, as interfaces desses contratos, na generalidade dos casos, com textos longos, complexos e difíceis de usar, são confeccionadas sem uma compreensão das necessidades de comunicação das partes envolvidas e da forma como elas interagem e interpretam essas informações. Este artigo, inspirado na Design Science Research (pesquisa da ciência do projeto), busca os fundamentos do design de informação e de interação presentes na literatura, sintetizando e organizando Proposições de Design que possam constituir um artefato para apoiar soluções de problemas na compreensão dos contratos eletrônicos, tomando como caso um site de reservas de hospedagens.

Palavras-chave: Visualização de Contratos, Contratos Visuais, Design de Informação, Design de Interação.

\begin{abstract}
Comercial relationships took on new characteristics since the rise of the internet, which enabled virtual commerce through electronic contracts as mediators of rights and obligations between users and companies. The recommendations for social isolation due Covid-19's pandemic increased the e-commerce. However, the interfaces of these contracts, in most cases, with long pages of texts, complex and difficult to use, are made without an understanding of the communication needs of the parties involved and the way they interact and interpret that information. This article searches fundamentals of information and interaction design and, inspired in the Design Science Research, synthesizes and organizes Design Propositions from literature that can constitute an artifact to solve problems that occur in the understanding of electronic contracts, taking as case a hotel booking sites.
\end{abstract}

Keywords: Contracts Visualization, Visual Contracts, Information Design, Interaction Design. 


\section{Introdução}

O comércio eletrônico vem crescendo diante da expansão de mercados consumidores em escala mundial, da exigência de rapidez nas negociações, da possibilidade de menores custos operacionais e, sobretudo, do momento atual da pandemia causada pelo novo coronavírus ${ }^{1}$ e as recomendações de isolamento social.

As relações comerciais, ainda que virtuais, são resguardadas por contratos, que correspondem ao acordo de vontades entre duas partes sobre um objeto economicamente apreciável. Apesar da importância dos contratos para proteção de direitos e garantia das obrigações, na prática, eles costumam ser extensos, complexos e de difícil compreensão, possuindo uma visualização pouco convidativa para seus destinatários, que, na maioria das vezes, são leigos em assuntos jurídicos (PASSERA; HAAPIO; BARTON, 2013). Nos negócios realizados por meio eletrônico, a função do contrato é ainda mais renegada. Uma pesquisa desenvolvida pela Universidade de Stanford constatou que 97\% dos usuários clicam no "concordo" sem ler os termos de uso (ROMERO, 2012). Não obstante, frente ao avanço tecnológico, esperava-se que o comércio eletrônico oferecesse ao consumidor uma comunicação mais efetiva e com menos percalços (SCHREIBER, 2014).

Para resolver tais questões, a Visualização de Contratos, um campo emergente, principalmente, na Europa Central (HAAPIO, 2013), utiliza fundamentos do design de informações e de interação em soluções práticas capazes de tornar os contratos mais claros e fáceis de usar, dotando as empresas de instrumentos para uma atuação não apenas corretiva, para resolver ações judiciais, mas, sobretudo, preventiva, garantindo um bom relacionamento com os consumidores. Consideram-se os contratos eletrônicos voltados à reserva de acomodações um caso de estudo em potencial, tendo em vista o número de usuários que usam a Internet para efetuar suas reservas, já que a presença física no local é usualmente impossibilitada pela distância entre os contratantes, bem como pelo alto custo de reservas via telefone ${ }^{2}$. Somado a isso, tem-se a complexidade dessa relação jurídica, porquanto a venda é realizada pela agência, mas o objeto do contrato é oferecido pelo hotel, além das dificuldades de comunicação em viagens internacionais.

Portanto, este artigo investiga como o design de informação e o de interação auxiliam na prescrição de uma linguagem gráfica e do uso de recursos de interação, para apoiar soluções de problemas de informação nos contratos eletrônicos, resultando na construção de um artefato que pode ser adotado como orientação em projetos de sites agenciadores de hotéis.

\footnotetext{
${ }^{1}$ O coronavírus é uma família de vírus que causa infecções respiratórias. Em março de 2020, foi declarada pandemia pela OMS. Disponível em:<https://coronavirus.saude.gov.br/>. Acesso em 25 mar. 2020 .

${ }^{2}$ No relatório Webshoppers (DIAS, 2016), a categoria "Viagens e Turismo" obteve a primeira colocação dos canais on-line que os consumidores mais utilizaram para realizar suas compras.
} 


\section{Metodologia}

A base metodológica deste trabalho se inspira na Design Science Research, pesquisa da ciência de projeto, ou seja, o corpo de conhecimento gerado sobre os artefatos e seu processo generativo para solução de problemas. Trata-se da ciência capaz de prescrever proposições de design para desenvolver e testar alternativas de soluções para problemas reais, introduzida por Herbert Simon, em 1969 (OLIVEIRA, 2010; DENYER et al., 2008; DRESH et al., 2015).

Segundo Perez et al. (2020), a Design Science permite ao design empregar suas próprias perspectivas metodológicas, sendo importante para a ampliação do campo epistemológico do design e das possibilidades de atuação dos designers, tendo sido utilizada nesta pesquisa justamente com o escopo de ampliar a atuação dos designers em projetos de contratos eletrônicos.

Entende-se por proposição de design um conjunto de intervenções, como normas gerais e bem fundamentadas, que podem ser usadas de acordo com a natureza específica do problema, possuindo boa probabilidade de solucionar o problema (DENYER et al., 2008; OLIVEIRA, 2010).

O método adotado nesta pesquisa contém as seguintes etapas: identificação dos problemas dos contratos eletrônicos; revisão sistemática da literatura, identificação e proposição de artefatos que investigam a fundamentação teórica da visualização de contratos e do design de informação e de interação, sintetizando em proposições de design para projetos de contratos eletrônicos; e, por fim, projeto e desenvolvimento do artefato, que estrutura e organiza as proposições de design, no caso de plataformas de reserva de acomodações.

Para mapear a produção acadêmica sobre a visualização de contratos, foi realizada uma pesquisa publicada pelo autor a partir das palavras-chave: 'visual contract' e 'contract visualization', nas bases de dados Web of Science, Science Direct, Scopus e Scielo, para publicações em inglês, e nas bases Portal de Periódicos Capes, Ibict, para publicações em português. Buscaram-se, também, resultados nas plataformas Google e Google Academics.

\section{Contratos eletrônicos e problemas no caso de reservas de hotéis}

Os contratos eletrônicos estabelecem direitos e deveres entre contratantes via Internet, mas não vêm respondendo claramente às referências - quem, onde, quando, como e o quê contrata utilizadas pelo direito contratual (SCHREIBER, 2014).

Isto é, usualmente, os contratantes têm informações inconsistentes sobre quem é a pessoa jurídica responsável, onde se localiza, ficando incertos sobre o local onde serão julgadas eventuais divergências, além de possuírem dúvidas sobre quando o contrato é fechado. Somamse a isto a forma como os contratos são incluídos nas interfaces com textos longos, complexos e de difícil entendimento e a própria prerrogativa de rapidez da Internet, que leva os consumidores a realizarem pesquisas superficiais sobre produtos ou serviços, sem saberem, muitas vezes, o que estão contratando. 
Além disso, cabe destacar a diferença entre o contrato que é firmado no e-commerce e os termos e condições de uso que os sites normalmente apresentam.

As especificações relativas ao produto ou serviço costumam aparecer nas páginas dedicadas às ofertas, já representando a definição do objeto do contrato. Geralmente, as preocupações com a apresentação gráfica se concentram nessas páginas, que fazem uso de fotografias, ícones, tabelas e outros recursos como forma de estimular a compra. Quando o consumidor insere o produto no carrinho de compras (telas de finalização da compra), outras informações podem ser acrescentadas, como prazo e as formas de pagamento.

Enquanto os termos e as condições de uso, usualmente disponibilizados nos rodapés dos sites e/ou próximos ao botão de finalização da compra, correspondem a cláusulas genéricas textuais, sem especificar o produto ou serviço que será contratado, incluindo informações sobre a empresa e seus fornecedores, regras gerais de uso do site, procedimentos para cancelamento do pedido, pagamento e entrega, comunicação com a empresa, responsabilidades, foro etc.

No caso dos contratos de sites de reserva de hotéis (ou de qualquer tipo de site que agencia outras empresas), essas relações comerciais ainda possuem o agravante da inclusão de informações específicas das condições da reserva e políticas do hotel, uma terceira parte envolvida nesses contratos. Essas condições da reserva dizem respeito às regras das acomodações e são acessadas nas páginas com dados sobre o produto e/ou serviço (com informações da acomodação em si), durante a finalização da compra, muitas vezes, em links e pop-ups específicos e, também, em e-mails de confirmação.

Assim, observa-se que as informações contratuais encontram-se dispersas e misturadas em várias páginas e links durante todo o processo da contratação eletrônica, exigindo uma leitura atenta do consumidor, para não deixar de acessar dados importantes, conforme a figura 1 .

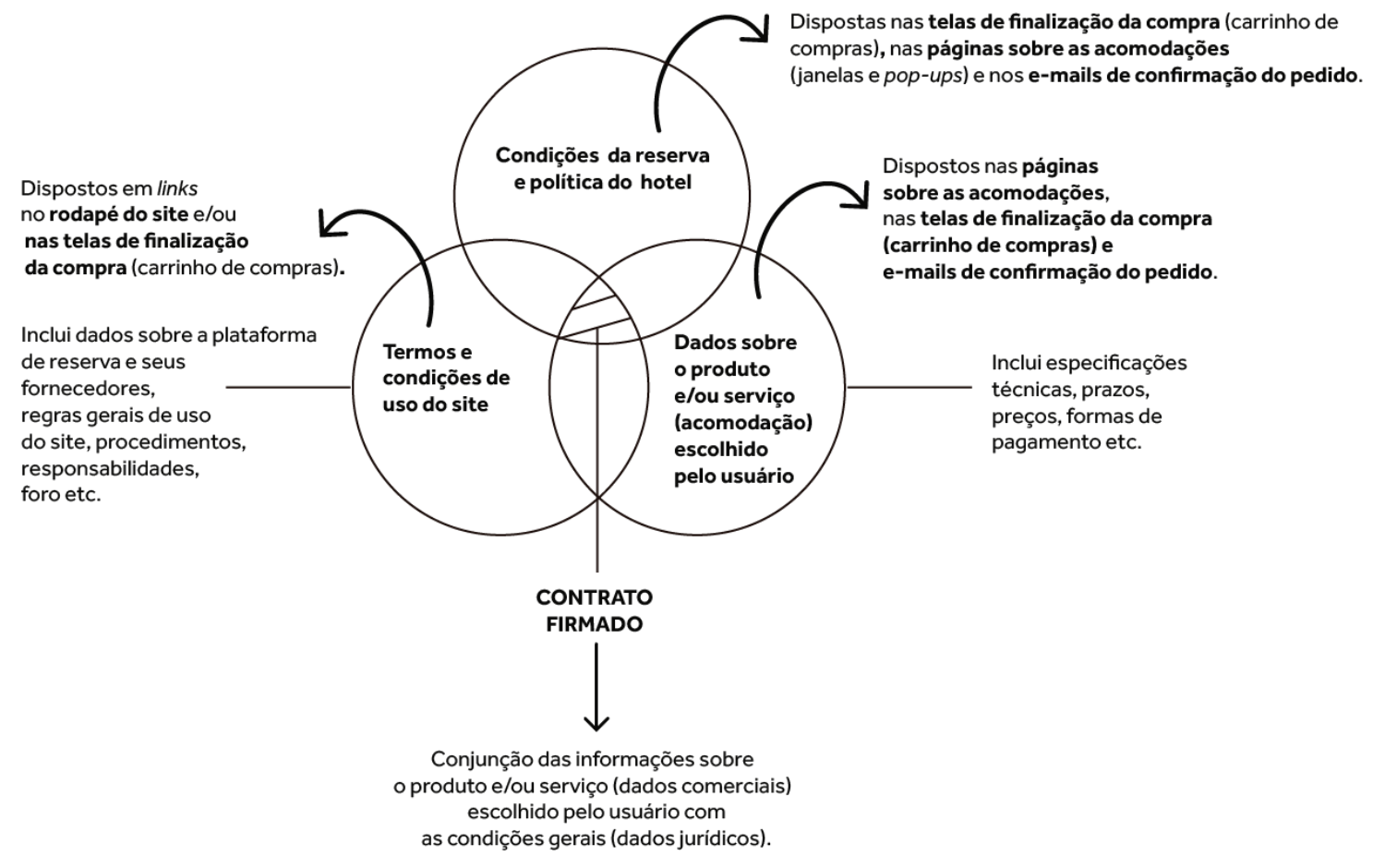

Figura 1: Contrato em sites de reserva de hotéis (autor) 
O contrato firmado pode ser compreendido a partir desses termos visíveis, que correspondem à parte explicitamente documentada (nas páginas de ofertas, nos termos e condições de uso dos sites, no e-mail de confirmação do pedido e/ou outros e-mails trocados etc.), mas também a partir de termos invisíveis, que são condições implícitas (legislação obrigatória, regras de inadimplência e práticas entre as partes, omissas no contrato), não estipuladas no contrato, mas que também se aplicam (NYSTÉN-HAARALA et al., 2010; HAAPIO, SIEDEL, 2013).

Embora as telas de ofertas e de finalização do pedido (carrinho de compras) já contenham informações do contrato, na maioria dos casos, a apresentação gráfica das cláusulas dos contratos eletrônicos não é incluída nas discussões dos projetos de e-commerce, resultando na dispersão das informações, na dificuldade de acesso às condições gerais, habitualmente, dispostas em locais de pouca visibilidade nas interfaces. Também não vem sendo apresentada a síntese do contrato para o usuário, que inclua tanto as informações do pedido, quanto as informações sobre os termos do site e outras condições implícitas importantes.

Frise-se que o consumidor, usualmente, não possui a compreensão de que este momento de pesquisa do produto e/ou serviço e sua inserção no carrinho de compras representa uma primeira etapa do processo contratual, razão pela qual ignora a leitura dos termos e condições de uso, concentrando sua atenção, basicamente, nas especificações do produto ou serviço a ser contratado.

A publicação apresentada por Marzullo et al. (2017) sobre a sequência da interação em um site de reservas apontou que a maioria dos entrevistados teve dificuldades de encontrar as informações sobre condições e políticas do hotel, achando que deveriam ser mais objetivas, enquanto outros sequer clicam nessa opção e tampouco nos termos gerais do site.

Após o momento do aceite da proposta, com o clique no botão de compra ou reserva, o contrato começa a ser executado (pagamento, entrega do produto/prestação do serviço contratado etc.) e os problemas costumam aparecer, tanto por desistência do consumidor diante de expectativas não realizadas e má gestão, quanto pela inadimplência do acordo por parte da empresa ou de seus fornecedores. O usuário, diante desses problemas, busca se informar sobre os termos e as condições que não ficaram evidenciados em todo o processo de contratação e/ou estabelecem contato com o suporte das empresas, na tentativa de compreender os termos visíveis e invisíveis e sanar suas dúvidas, podendo resultar em divergências de interpretações das cláusulas, reclamações em canais de reputações de empresas de e-commerce e, até mesmo, ações judiciais.

A compreensão da contratação eletrônica, do ponto de vista de um processo, permite notar a importância da comunicação das informações, tanto nas etapas pré-contratuais (elaboração e comprometimento), quanto nas etapas de execução do contrato. Embora as empresas realizem investimentos visando à construção de plataformas digitais, os contratos eletrônicos, geralmente, não fazem parte das discussões desses projetos, a despeito de seu potencial para contribuir em relações comerciais transparentes.

Embora esses problemas relacionados à visualização dos contratos tenham sido identificados no caso específico de reserva de hotéis, eles constituem uma classe de problemas comuns na contratação eletrônica. 


\section{Proposições de Design e Proposta do Artefato}

Os referenciais teóricos desta pesquisa se baseiam na convergência do design de informação e de interação, por compartilharem preocupações sobre as necessidades dos usuários, ficando a Visualização de Contratos na interseção desses dois campos de pesquisa.

Assim, para minimizar os problemas dos contratos eletrônicos, propõe-se repensar como são elaborados e sua apresentação gráfica, configuração e usabilidade, a partir dessas disciplinas, decompostas, com base no framework de Twyman (1985) (Quadro 1), em proposições de design:

\begin{tabular}{l|l}
\hline Conteúdo informacional & Framework de Twyman (1985): \\
\hline Modo de simbolização & $\begin{array}{l}\text { quais as linguagens (verbal, pictórico, esquemático ou a mistura } \\
\text { de dois ou mais) que melhor atingem aquele objetivo; }\end{array}$ \\
\hline Configuração & $\begin{array}{l}\text { qual a melhor organização dos elementos gráficos no espaço } \\
\text { para alcançar o propósito desejado; }\end{array}$ \\
\hline Propósito & qual é o objetivo daquela mensagem; \\
\hline Usuários & $\begin{array}{l}\text { considerar as necessidades de informação dos usuários levando em } \\
\text { consideração fatores como idade, habilidades, treinamento, interesses e } \\
\text { experiência anterior, etc; }\end{array}$ \\
\hline Circunstâncias de uso & $\begin{array}{l}\text { qual é o contexto em que o usuário interage com esta informação; } \\
\text { Quadro 1: Framework de Twyman (1985) }\end{array}$
\end{tabular}

\section{Conteúdo informacional}

O conteúdo dos contratos eletrônicos pode ser dividido em dados jurídicos (termos e condições de uso), tais como identificação das partes, reponsabilidades, planejamento de contingências, riscos, foro e métodos para resolução de litígios caso haja algum impasse, regras para o cancelamento e dados do negócio (escolha do usuário nas páginas com dados sobre o produto ou serviço e suas condições), tais como escopo, objeto, prazos, entregas, cronograma, formas de pagamento, valores e procedimentos (PASSERA, HAAPIO, BARTON, 2013; HAAPIO, 2013). Esses dados costumam ser apresentados de forma separada, sobrecarregando cognitivamente os usuários (Figura 2). 


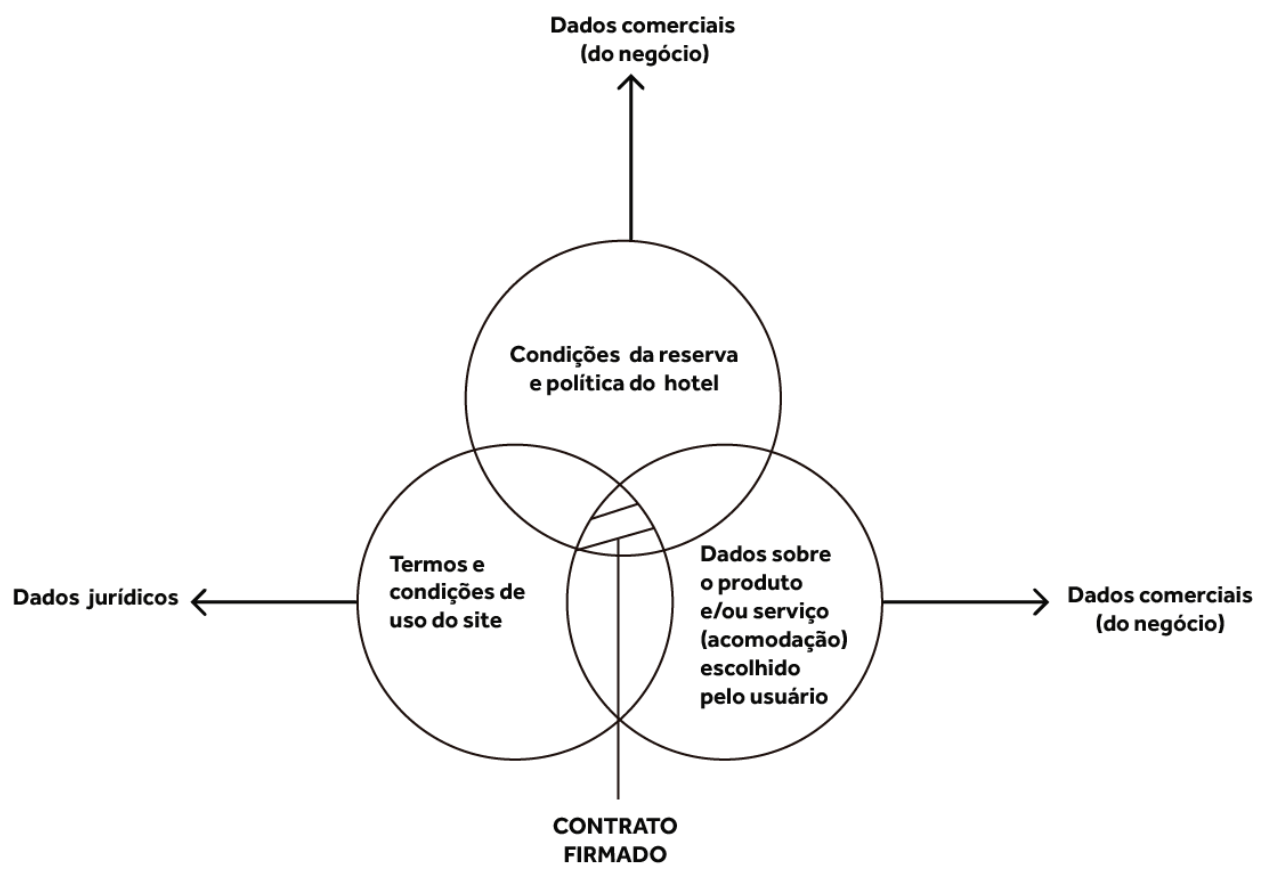

Figura 2: Dados brutos do contrato eletrônico (autor)

Considerando esses dados, que podem se transformados em informações para produzir conhecimento, tem-se a primeira PD:

PD 1 Identificação do conteúdo informacional

- Identificar os dados (jurídicos e comerciais), com o objetivo de transformá-los em informações úteis, unificadas e completas (HAAPIO, PASSERA, 2013b; PASSERA, HAAPIO, CURTOTTI, 2014).

Após esse reconhecimento, faz-se importante a estruturação, como sugere a segunda PD:

PD 2 Organização dos conteúdos

- Organizar os conteúdos a partir das respostas às perguntas: quem?, o quê?, onde?, quando?, como?, por quê? e o que acontece se fizer ou se não fizer? (HAAPIO, 2013; SCHREIBER, 2014).

- Organizar os conteúdos pelo tipo de informação: espacial, cronológica, quantitativa e narrativa-descritiva (RAJAMANICKAN, 2005; LIMA, 2009).

\section{Modo de simbolização}

O modo de simbolização trata do uso estratégico das linguagens visuais gráficas verbal, pictórica e esquemática (TWYMAN, 1985; LIMA, 2009) que melhor se adequem à informação 
que se deseja transmitir, de acordo com seus usuários e seu contexto de uso (PASSERA, HAAPIO, BARTON, 2013).

$\mathrm{Na}$ maioria dos casos, não ocorre adequação significativa nos contratos para o meio eletrônico, priorizando o uso da linguagem verbal. Por isso, a visualização de contratos visa expandir o uso de outras linguagens, objetivando a comunicação clara e efetiva, conforme a PD 3:

PD 3 Uso das linguagens gráficas verbal, pictórica e esquemática de forma complementar

- Priorizar as linguagens visuais gráficas que melhor comuniquem o conteúdo contratual, de acordo com o contexto de uso do meio eletrônico e das necessidades do usuário (TWYMAN, 1985; LIMA, 2009; PASSERA, HAAPIO, BARTON, 2013).

Essas linguagens podem ser usadas segundo as PD 4, 5 e 6 :

PD 4 Simplificação, organização e estruturação da linguagem verbal

- Identificar as informações principais e secundárias do contrato, hierarquicamente. Escrever textos curtos, concisos, objetivos e escaneáveis (SCHADE, 2018; LIMA, 2009).

- Usar textos em primeira pessoa (WALLER et al., 2016).

- Usar menus de navegação, cabeçalhos pesquisáveis, destaques tipográficos e listas com marcadores, para aumentar a leiturabilidade e legibilidade na web (HAAPIO, PASSERA, 2016; WALLER et al., 2016).

PD 5 Uso da linguagem pictórica

- Compor a partir das variáveis forma, tamanho, valor, orientação posição, textura e cor (BERTIN, 2011), através dos recursos: enfatizar ou minimizar; comparar ou ordenar; agrupar ou classificar; selecionar ou omitir; optar pelo reconhecimento imediato ou tardio; apresentar de forma interessante; hierarquizar, ligar ou separar, igualar ou diferenciar etc. (MIJKSENAAR, 1997; BONSIEPE, 1999; WALLER et al., 2016).

- Criar relações significativas entre objetos e espaços gráficos (ENGELHARDT, 2002).

- Usar fotografias, desenhos ou representações figurativas e literais para a identificação de informações tangíveis (BARTON et al., 2013; HAAPIO, PLEWE, ROOY, 2017).

- Usar imagens semi-figurativas (esquemáticas) sintéticas, como ícones (literais ou não de acordo com a informação), para a representação pictórica de informações intangíveis (BARTON et al., 2013; LIMA, 2009; HAAPIO, PASSERA, 2016). 
PD 6 Uso da linguagem esquemática

- Compor a partir das variáveis forma, tamanho, valor, orientação posição, textura e cor (BERTIN, 2011), através dos recursos: enfatizar ou minimizar; comparar ou ordenar; agrupar ou classificar; selecionar ou omitir; optar pelo reconhecimento imediato ou tardio; apresentar de forma interessante; hierarquizar, ligar ou separar, igualar ou diferenciar etc. (MIJKSENAAR, 1997; BONSIEPE, 1999; WALLER et al., 2016).

- Criar relações significativas entre objetos e espaços gráficos (ENGELHARDT, 2002).

- Buscar a excelência dos gráficos (TUFTE, 2001, 2006).

- Usar diagramas, mapas, gráficos, tabelas, linhas do tempo (ENGELHARDT, 2002; RAJAMANICKAM, 2005; HAAPIO, PASSERA, 2016).

\section{Configuração}

Entende-se por configuração a organização dos elementos no espaço para alcançar o propósito desejado. Apesar da gama de possibilidades de configurações existentes, os contratos eletrônicos continuam sendo criados de forma linear, sendo interrompidos pelas margens dos sites e, em alguns casos, em lista, sem maiores questionamentos sobre a configuração mais adequada.

A visualização de contratos, ao expandir o uso de outros modos de simbolização, estabelece configurações mais dinâmicas, que possibilitam maior liberdade à leitura e ajudam, inclusive, os usuários que apenas escaneiam as telas digitais (leitura de partes do texto, através de palavraschave e elementos de quebra) (HAAPIO, HAGAN, 2016).

Assim, com relação à configuração, tem-se a PD 7:

PD 7 Configurações mais dinâmicas que suportem a leitura escaneável

- Expandir o uso dos modos de simbolização pictórico e esquemático em contratos, através das configurações linear puro, linear interrompido, lista, linear ramificado, matriz, não linear dirigido e não linear aberto (TWYMAN, 1979).

- Configurar páginas através de índices, menu de navegação, resumo do contrato e sumários (HAAPIO, HAGAN, 2016).

\section{Propósito}

Refere-se aos objetivos da mensagem que podem ser combinados e reordenados nos contratos eletrônicos, desde que a provisão de informações e instrução seja sempre o critério prioritário (TWYMAN, 1985). 
PD 8 Uso das linguagens com o propósito de oferecer informação adequada à comunicação e instrução

- Estabelecer prioridades entre as funções: descrição, narração, persuasão e exortação, instrução e provisão de informações, dar prazer ao leitor, desde que a provisão de informações e instrução seja o critérios prioritário (TWYMAN, 1985).

- Definir prioridades entre os objetivos comunicativos da linguagem esquemática (narração, instrução, exploração, simulação) no contrato eletrônico, principalmente em oferecer instruções (RAJAMANICKAN, 2003; BARTON et al., 2013; WALLER et al., 2016; PASSERA, 2018).

\section{Usuários}

Para que os contratos realmente funcionem e alcancem seus propósitos, precisam ser projetados, especialmente, para os usuários que realizam compras online.

PD 9 Identificação das necessidades de informação e interação dos usuários para aprimorar a experiência

- Garantir que os contratos atendam às necessidades de usuários não advogados (HAAPIO, PASSERA, 2013a).

- Identificar aspectos indesejáveis e desejáveis da experiência dos usuários com contratos eletrônicos (PREECE et al., 2013; HAAPIO, PASSERA, 2013a; HAAPIO, BARTON, 2017; HAAPIO, PASSERA, 2016).

\section{Circunstâncias de uso}

Parte do processo de entender os usuários, identificando suas necessidades e os aspectos da experiência, consiste em considerar o contexto em que interagem com os contratos eletrônicos. Assim, a identificação dos fatores emocionais envolvidos durante a interação permite investigar, também, questões relacionadas à usabilidade e ao desempenho, que influenciam a experiência e a percepção dos usuários.

PD 10 Identificação das circunstâncias de uso para assegurar eficiência, eficácia e satisfação

- Operacionalizar as metas de usabilidades: eficácia, eficiência, segurança, utilidade, learnability e memorability (PREECE et al., 2013).

- Refletir sobre os princípios do design de interação durante o projeto: Visibilidade; Resposta do sistema; Restrição; Consistência; Potencialidade; Mapeamento (PREECE et al., 2013; CAIRO, 2008a e 2008b). 
- Aplicar as heurísticas de Nielsen (1995): Visibilidade do status; Correspondência entre o sistema e realidade; Controle do usuário; Consistência e padrões; Prevenções de erros; Reconhecimento; Flexibilidade e eficiência de uso; Estética minimalista; Auxílio a recuperação de erros; Ajuda e documentação.

- Usar objetos de interação (MIRANDA, 2013; ANDRADE, 2014).

Essas proposições de design identificadas na revisão da literatura podem ser estruturadas e organizadas de modo a facilitar a adoção de soluções para problemas inerentes à visualização e à compreensão de contratos eletrônicos. Dessa forma, preconiza-se um artefato como instrumento de apoio à solução dessa classe de problemas (Figura 3).

\section{PROPOSTA DEARTEFATO PARA PROJETOS DE CONTRATOS ELETRÔNICOS VISUAIS}

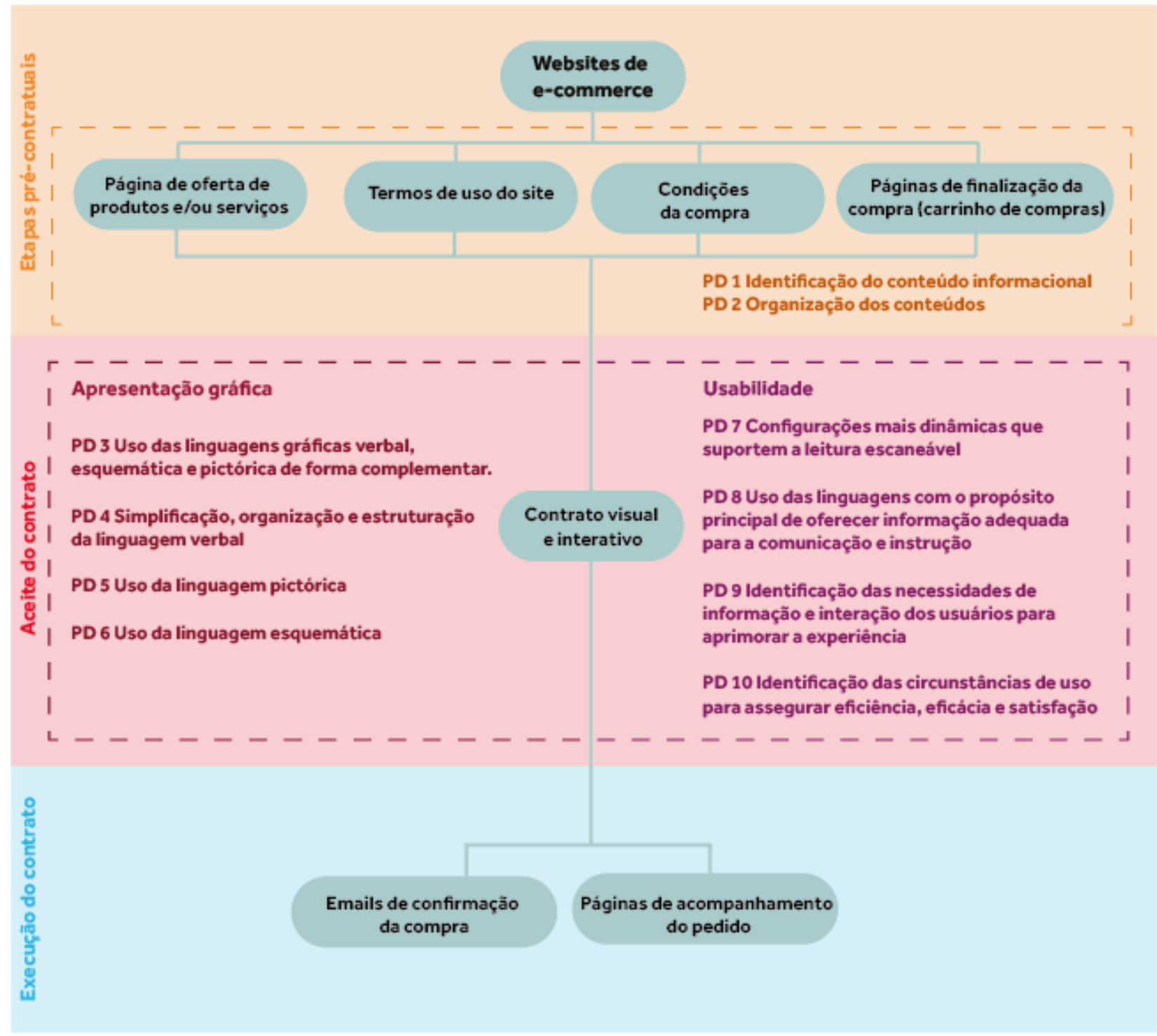

Figura 3: Proposta de artefato para projetos de contratos eletrônicos visuais e interativos (autor) 
Nele, identificam-se os pontos de contato dos usuários em websites de e-commerce que incluem informações contratuais e são passíveis de serem projetados por designers, divididos pelas etapas do processo de contratação online, servindo como guia sobre uso das proposições de design para a Visualização de Contratos eletrônicos.

Enfatiza-se a importância de evidenciar o momento de aceite do acordo, através de um contrato visual e interativo, como forma de minimizar os problemas das contrações eletrônicas.

A seção a seguir apresenta uma proposta de aplicação do artefato e suas proposições de design no caso real de sites de reserva de hotéis.

\section{Proposta de Aplicação do Artefato}

A identificação e organização dos dados jurídicos e de negócio (PD 1 e 2) dispersas nas páginas das acomodações, de finalização da reserva, de termos de uso do site e em pop-ups com condições de reserva, evidenciaram a necessidade de desenvolver uma etapa no processo de contratação on-line, com o objetivo de sintetizar as implicações comerciais e jurídicas da relação a ser estabelecida. Essa etapa corresponde ao momento de aceite do contrato (Figuras 4 e 5), permitindo ao usuário a revisão das informações selecionadas antes de finalizar a compra, minimizando a chance de erros (PD 8, 9 e 10).

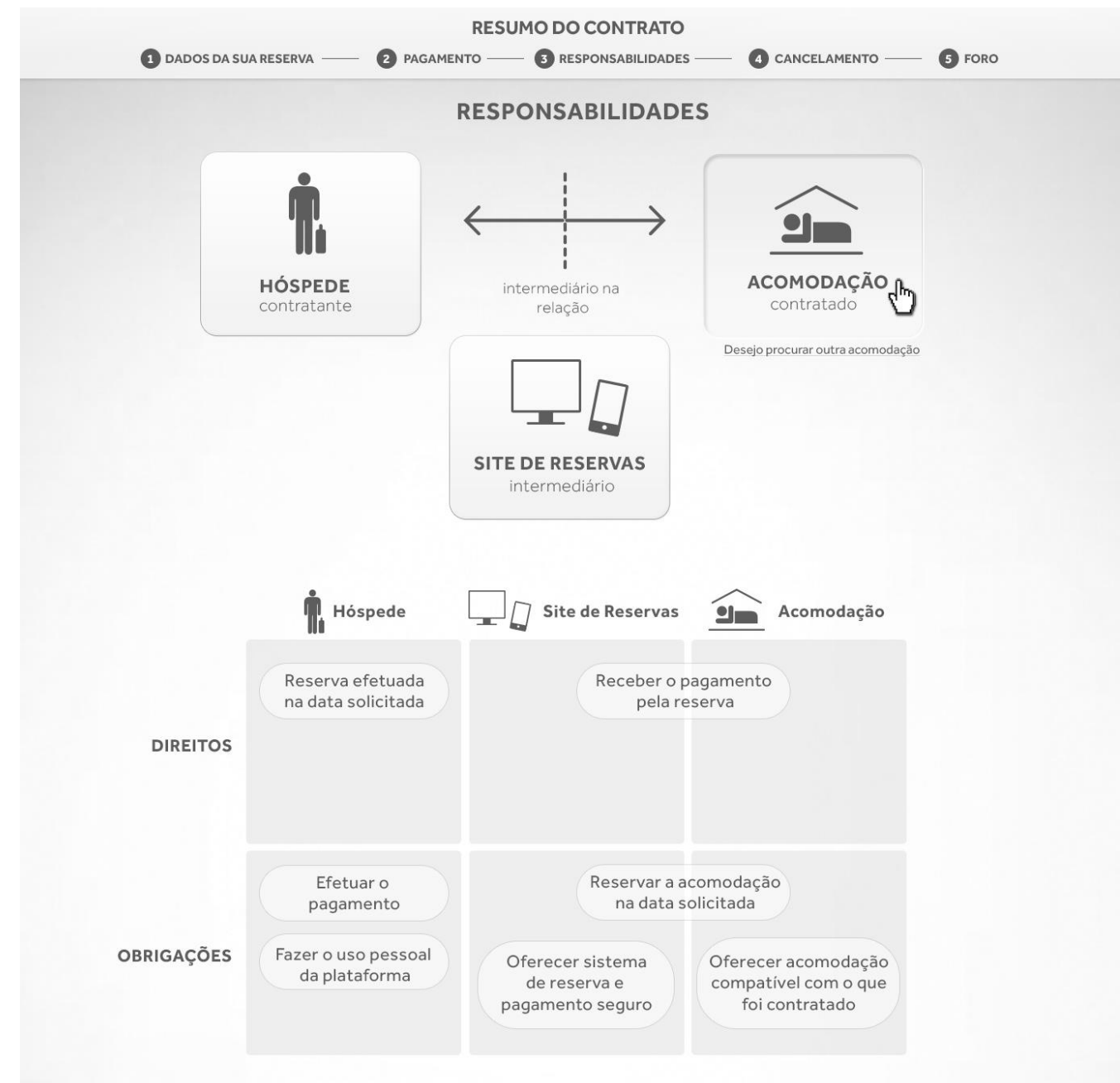


CANCELAMENTO

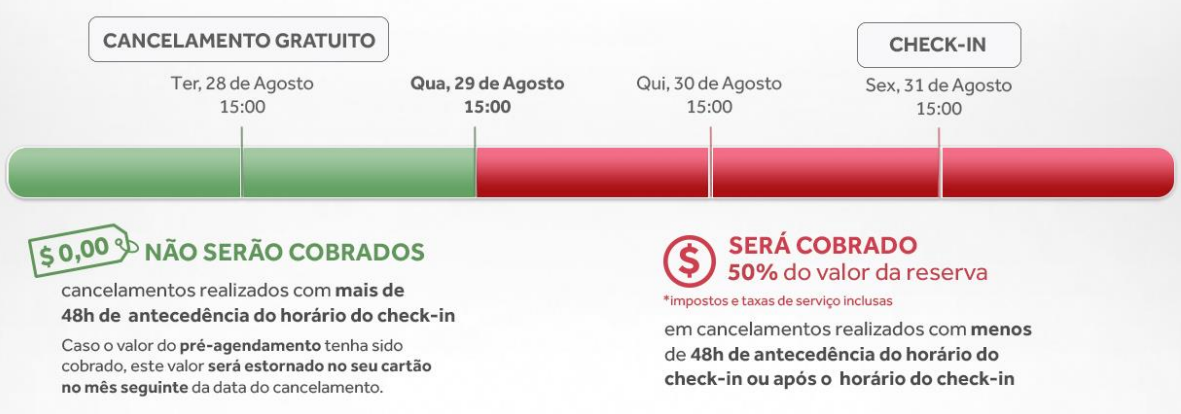

COMO PROCEDER?
(1) Sempre comunique ao hotel sobre atrasos no check-in ou se for chegar no dia seguinte.
(2) Utilize o link da confirmação da sua reserva ou entre no site de reservas

(3) Negociação sobre tarifa de cancelamento para cancelar. diretamente com a acomodação.

\section{OUTRAS SITUAÇÕES}

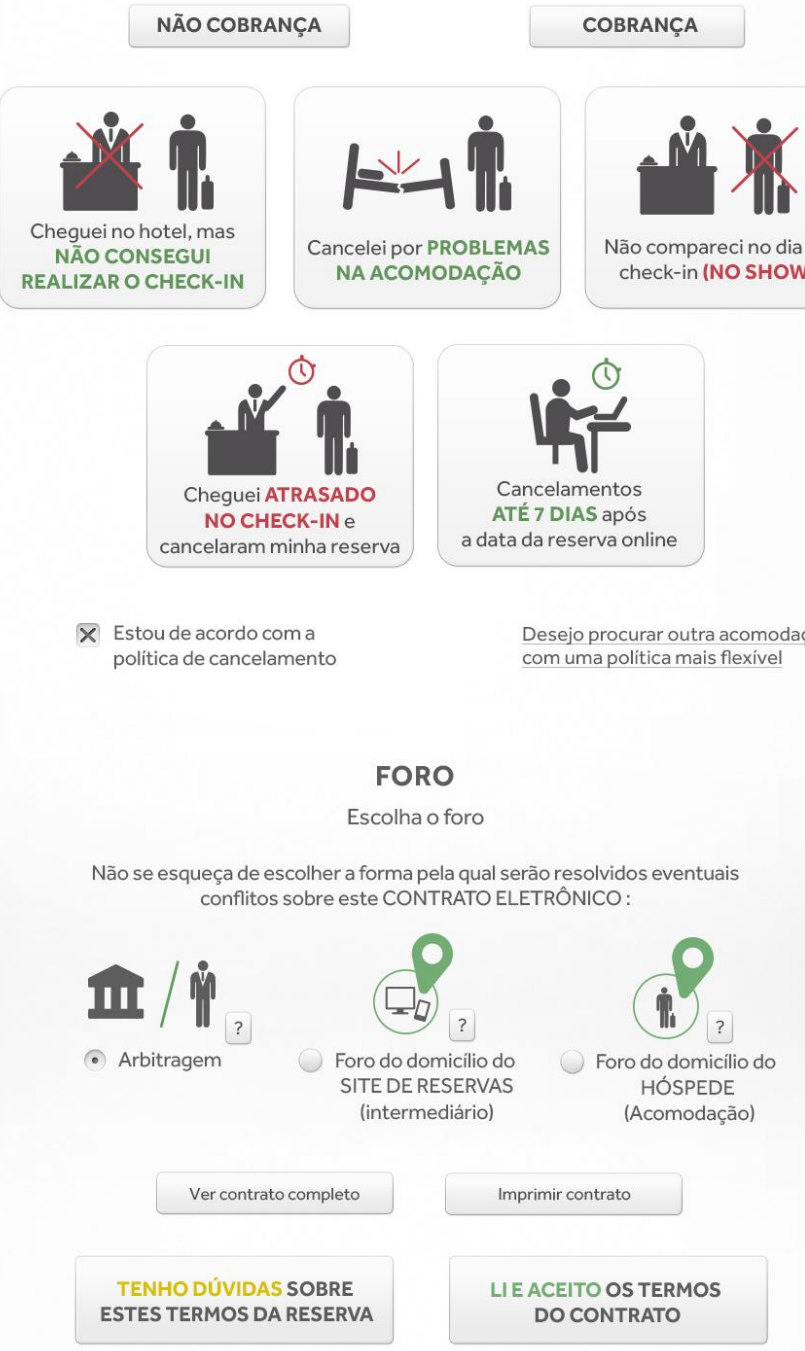

Figura 5: Continuação da síntese do contrato em One page layout (autor) 
Optou-se por uma configuração em one page layout (PD 7), com as informações principais simplificadas em uma única tela (PD 4), que pode ser navegada através de um menu com links internos e ícones (PD 5), apresentados em formato de diagrama (PD 6), correspondentes à sequência das seções do contrato (Figura 6), permitindo ao usuário acompanhar o progresso da leitura (PD 8 e 9).

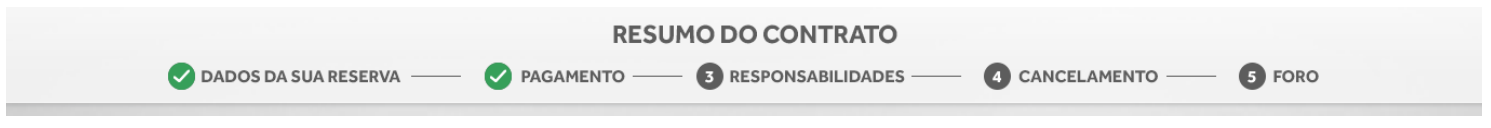

Figura 6: Menu de navegação em formato de diagrama (autor)

O contrato fez uso das linguagens verbal (simplificada), pictórica e esquemática de forma complementar (PD 3) e as informações secundárias foram incluídas em subpáginas através de botões e menus interativos que abrem janelas (popups) em tela cheia (PD 10).

Foram utilizados ícones (PD 5) para identificar os contratantes, situações e valores do cancelamento dispostos em infográficos (PD 6) interativos e menus (PD 10) - diagrama para identificação das partes; tabela para entendimento das responsabilidades; linha do tempo codificada pelas cores vermelho e verde indicando o prazo para cancelamento; menu interativo para apresentar a política de cancelamento, com filtros codificados pelas cores vermelho para as situações de cobrança e verde para as de não cobrança; diagrama passo a passo para mostrar os procedimentos para efetuar o cancelamento - que buscaram a excelência dos gráficos ao evidenciar os relacionamentos estabelecidos.

Merecem destaque as cinco narrativas (PD 8) em primeira pessoa (PD 4) de situações adversas reais criadas para a identificação rápida do usuário (Figura 7) que esteja sob estresse e precise cancelar e a inclusão de uma caixa de seleção que restringe os usuários a declararem ter lido, como forma de mostrar a relevância do entendimento desta cláusula, mesmo para um leitor desatento (PD 9).

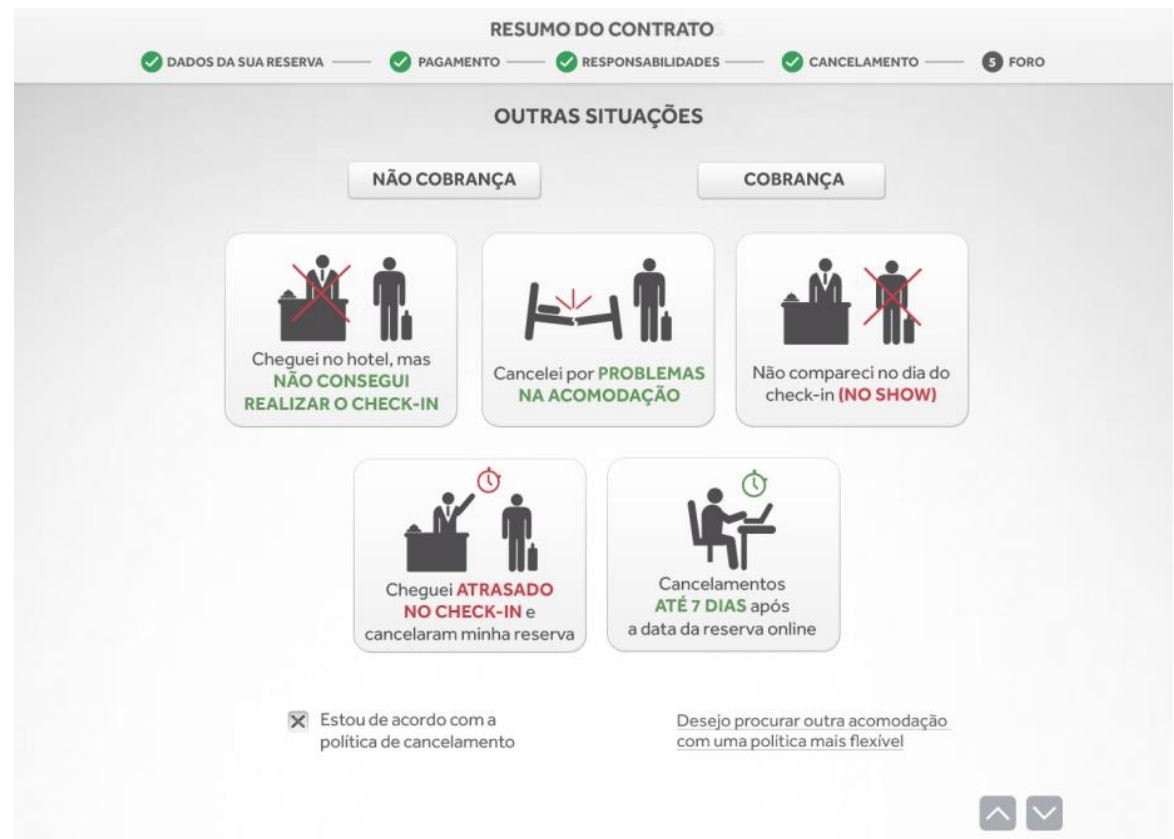

Figura 7: Menu interativo com as situações de cancelamento (autor) 
Ressalta-se, ainda, que foi utilizado um formulário de botão de rádio (radio button) na cláusula sobre o foro (Figura 8), possibilitando ao usuário escolher onde serão julgadas eventuais ações judiciais (PD 9 e 10).

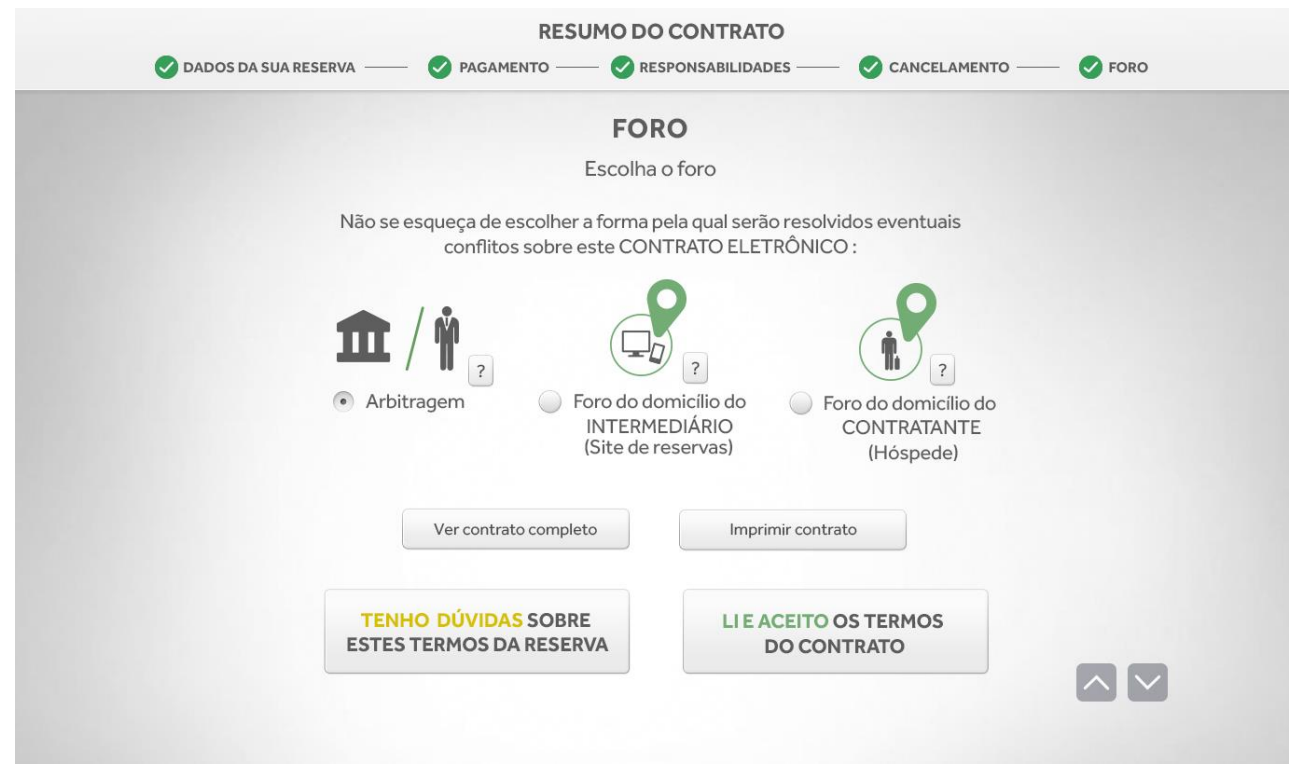

Figura 8: Formulário fazendo uso de ícones (autor)

\section{Considerações finais}

Ainda que sejam necessários testes com usuários para avaliar a compreensão dessas informações visuais e esquemáticas apoiadas por recursos de interação - em comparação aos contratos eletrônicos tradicionais -, os primeiros resultados sugerem que o e-commerce poderia se beneficiar de uma abordagem mais visual e interativa para a apresentação de seus termos, tanto do ponto de vista do consumidor quanto das empresas.

Destaca-se o potencial da Design Science Research como abordagem metodológica adequada para estruturar e organizar conceitos de diferentes áreas de pesquisa, convergindo para artefatos que possuem potencial para a solução de determinada classe de problemas. Ressaltamse as contribuições de natureza prática para projetos de contratos eletrônicos, questão que ganha relevância, tendo em vista a expansão do e-commerce diante das recomendações de isolamento impostas pela pandemia da COVID-19. Além disso, a ampliação das discussões sobre a fundamentação teórica do design de informação e de interação é relevante para que pesquisas futuras desenvolvam uma base sólida para o campo da Visualização de Contratos.

Esse debate, contudo, não se restringe ao design, considerando que a aplicação dos fundamentos teóricos propostos neste trabalho pode, inclusive, produzir algum impacto na jurisprudência consagrada nas Cortes Judiciárias, como, por exemplo, a utilização de formulário (na cláusula do foro) para escolha e interação direta com o usuário, circunstância capaz de modificar a própria natureza do contrato de adesão. 
Por fim, espera-se que a pesquisa das visualizações de contratos possa dotar usuários e empresas de ferramenta de comunicação mais eficiente, que as conscientize e oriente quanto aos direitos e deveres, evitando-se ou, ao menos, reduzindo-se a quantidade de litígios virtuais.

\section{Referências}

ANDRADE, R. Infográficos animados e interativos em saúde. Dissertação (Mestrado em Design) - Universidade Federal do Paraná, Curitíba. 2014.

BARTON, T. et al. Visualization: seeing contracts for what they are, and what they could become. In: Journal of Law, Business \& Ethics, 19, p. 47-63, 2013.

BERTIN, J. Semiology of Graphics: diagrams networks maps. California: Esri Press, 2011.

BONSIEPE, G. Del objeto a la interfase: mutaciones del diseño. Buenos Aires: Ediciones Infinito, 1999.

CAIRO, A. Infografía 2.0. Madri: Alamut. 125 p. 2008 a.

Interactividad: la nueva frontera de la visualización de información en prensa. In: PEREZ, A; GIL, A. (eds.). $15^{\circ}$ Premios Internacionales de Infografía Malofiej. Pamplona: SNDE/Universidad de Navarra, 2008b.

DENYER, D. et al. Developing design propositions through research synthesis. Organization Studies 29. p. 393-413, 2008.

DRESH, A. et al. Design science research: método de pesquisa para avanço da ciência e tecnologia. Porto Alegre: Bookman, 2015.

DIAS, A. Relatório WebShoppers. In: E-bit. 33. Ed, 2016. Disponível em: <https://www.ebit.com.br/webshoppers>. Acesso em: 17 de jul. 2017.

ENGELHARDT, Y. The language of graphics. Amsterdam: Sewn, 2002.

HAAPIO, H. Contract clarity and usability through visualization. In: MARCHESE, F., BANISSI, E. Knowledge Visualization Currents - From Text to Art to Culture. SpringerVerlag London, cap. 4, 2013.

; BARTON T. Business-friendly contracting: how simplification and visualization can help bring it to practice. In: Jacob K., Schindler D., Strathausen R. (eds.) Liquid Legal. Management for Professionals. Springer, Cham. p. 371-396, 2017.

; HAGAN, M. Design Patterns for Contracts. In: E. Schweighofer, F. Kummer, W. Hötzendorfer \& G. Borges, eds., Networks. Proceedings of the 19th International Legal Informatics Symposium IRIS 2016. Wien: Österreichische Computer Gesellschaft OCG/books@ocg.at, p.381-8, 2016.

; PASSERA, S. Contracts as interfaces: exploring visual representation patterns in contract design. In: M. J. Katz, R. A. Dolin \& M. Bommarito (eds.) Legal Informatics, Cambridge, UK: Cambridge University Press. Published ahead of print as part of doctoral dissertation, 37 pages, 2016.

PASSERA, S. Transforming contracts from legal rules to usercentered communication tools. In: Communication Design Quarterly 1.3. Symposium on Communicating Complex Information, Greenville NC, USA, 2013a.

; PASSERA, S. What Lawyers Need to Learn from Information Designers? In:

Legal Information Institute. 2013b. Disponível em:


<https://blog.law.cornell.edu/voxpop/2013/05/15/visual-law-what-lawyers-need-to-learnfrom-information-designers>. Acesso em 26 jul. 2018.

; PLEWE, D.; ROOY, R. Contract continuum: from text to images, comics and code. In: Erich Schweighofer et al. (Eds.), Trends and Communities of Legal Informatics. Proceedings of the 20th International Legal Informatics Symposium IRIS 2017. Österreichische Computer Gesellschaft, Wien 2017, p. 411-418, 2017.

; SIEDEL, G. A short guide to contract risk. In: Gower Publishing. p. 232. 2013.

PEREZ, I. et al. A design science nas pesquisas em design no Brasil. In: Estudos em Design. Revista (online). Rio de Janeiro: v. 28. n. 1. p. 38-52, 2020.

PREECE, J. et al. Design de interação: além da interação homem-computador. Porto Alegre: Bookman, 2013.

LIMA, R. Análise da infografia jornalística. Dissertação (Mestrado em Design) ESDI/UERJ, Rio de Janeiro, 2009.

MARZULLO, R. et al. Analysis of users' mental model and expectations from usability and information design point of view in e-contracts. In: Springer International Publishing AG, 2017.

MIJKSENAAR, P. Visual function: an introduction to information design. New Jersey, Princeton Architectural Press, 1997.

MIRANDA, F. Animação e interação na infografia jornalística. Dissertação (Mestrado em Design). Universidade Federal do Paraná. Curitiba, 2013.

NIELSEN, J. Ten Usability Heuristics. In: Nielsen e Norman Group, 1995. Disponível em: <https://www.nngroup.com/articles/ten-usability-heuristics/>. Acesso em: 05 set 2017.

NYSTE'N-HAARALA, S. et al. Flexibility in contract terms and contracting processes In: International Journal of Managing. In: International Journal of Managing, Projects in Business, Vol. 3 No. 3, pp. 462-478, Emerald Group Publishing Limited, 2010.

OLIVEIRA, A. Uma avaliação de sistemas de medição de desempenho para P\&D implantados em empresas brasileiras frente aos princípios de construção identificados na literatura. Tese (Doutorado em Engenharia de Produção) - COPPE/UFRJ, Rio de Janeiro, 2010.

PASSERA, S. Why contracts need visualization? 2013. Disponível em: $<$ http://www.mindspace.fi/en/english-why-contracts-need-visualization/>. Acesso em 29 abr. 2018.

; HAAPIO, H.; BARTON, T. Innovating Contract Practices: Merging Contract Design with Information Design. In: Proceedings of the 2013 academic forum on integrating law and contract management, 2013.

; HAAPIO, H.; CURTOTTI, M. Making the Meaning of Contracts Visible:

Automating Contract Visualization. Erich Schweighofer et al. (Eds.). Transparency. Proceedings of the 17th International Legal Informatics Symposium IRIS 2014. Österreichische Computer Gesellschaft OCG, Wien 2014, p. 443-450. 2014.

RAJAMANICKAM, V. Infographics seminar handout, 2005. Disponível em: <www.as8.it/handouts/infographic.pdf>. Acesso em: 17 de jul. de 2017.

ROMERO, L. Não li e concordo, Super Interessante, 2012. Disponível em: $<\mathrm{http}$ //super.abril.com.br/tecnologia/nao-li-concordo-contratos-termos-sites-redes-sociais698482.shtml>. Acesso em: 22 de set. de 2014.

SCHREIBER, A. Contratos eletrônicos e consumo. Revista Brasileira de Direito Civil (Revista Eletrônica), Rio de Janeiro, v. 1, 2014. 
SCHADE. A. Inverted pyramid: writing for comprehension, 2018. Disponível em: <https://www.nngroup.com/articles/inverted-pyramid/>. Acesso em: 02 jul. 2018.

TUFTE, E. The visual display of quantitative information. 2 ed. Connecticut: Graphics Press, 2001.

Beautiful evidence. Connecticut: Graphics Press, 2006.

TWYMAN, M. A schema for the study of graphic language. Kolers, P.A. \& Wrostad, M.E. \& Bouma, H. (Eds.), In: The Processing of Visible Language, vol. 1, Plenum, New York, p. 117-150, 1979.

Using pictorial language: a discussion of the dimensions of the problem. In: T. M. 312 Dufty and R. Waller (eds.) Designing usable texts. Orlando, Florida: Academic Press, p. 245-312, 1985.

WALLER, R. et al. Cooperation through clarity: designing simplified contracts. In: Journal of Strategic Contracting and Negotiation. Vol. 2(1-2) p.48-68, 2016.

\section{Sobre os autores}

\section{Renata Zappelli Marzullo}

Possui graduação em Comunicação Visual Design pela Universidade Federal do Estado do Rio de Janeiro e mestrado na Escola Superior de Desenho Industrial da Universidade do Estado do Rio de Janeiro na linha de pesquisa Design e Tecnologia pesquisando os campos design da informação e de interação. Atualmente é programadora visual da Casa da Ciência - Centro Cultural de Ciência e Tecnologia da Universidade Federal do Estado do Rio de Janeiro.

0000-0001-8260-5924

\section{André Ribeiro de Oliveira}

Possui graduação em Engenharia Eletrônica pela Universidade Federal do Rio de Janeiro, mestrado e doutorado em Engenharia de Produção pela mesma instituição com área de concentração em Gestão e Inovação. Atualmente é Professor Associado do Departamento de Engenharia Industrial da Universidade do Estado do Rio de Janeiro e do Programa de PósGraduação em Design da Escola Superior de Desenho Industrial da mesma instituição. 0000-0003-2304-8288

\section{André Soares Monat}

Possui graduação em Engenharia de Infra Estrutura Aeronáutica pelo Instituto Tecnológico de Aeronáutica, graduação em Engenharia Elétrica, mestrado em Engenharia de Sistemas e Computação pela Universidade Federal do Rio de Janeiro /COPPE (Bolsista CNPq -1988) e doutorado em Engenharia de Sistemas e Computação - pela University of East Anglia, Reino Unido (Bolsista CAPES - 1993). Professor da Escola Superior de Desenho Industrial (ESDI/UERJ).

0000-0002-7985-5794 